\title{
Wo ist der Fortschritt?
}

\author{
Where is the Progress?
}

Autor

Institut
Stefan Priebe

Unit for Social and Community Psychiatry, Queen Mary University of London, Großbritannien

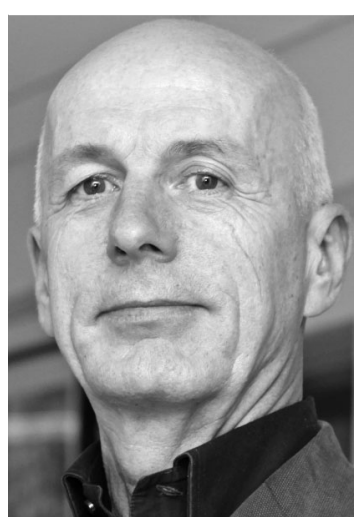

Prof. Dr. med. Stefan Priebe
Bibliografie

DOI http://dx.doi.org/

10.1055/s-0031-1298907

Psychiat Prax 2012; 39: 55-56

(c) Georg Thieme Verlag KG

Stuttgart · New York

ISSN 0303-4259

Korrespondenzadresse

Prof. Dr. med. Stefan Priebe

Unit for Social and Community Psychiatry, Newham Centre for Mental Health

London E13 8SP

United Kingdom

s.priebe@qmul.ac.uk
Wenn man die Geschichte in Generationen einteilt, zählt eine Generation normalerweise 30 Jahre. Die jetzt zu Ende gehende Generation begann also ungefähr 1982, die davor 1952. Welchen Fortschritt haben diese beiden Generationen der Psychiatrie beschert? Mit Fortschritt soll hier nicht neues Wissen gemeint sein - wie etwa Erkenntnisse über soziale und genetische Bedingungsfaktoren, biologische Korrelate von Störungen oder Wirkmechanismen von Therapien -, sondern allein die Entwicklung von effektiven Behandlungen für die Patienten, also das, was den Patienten in der täglichen Praxis wirklich zugute kommt und ihr Leiden lindert. Und angesichts der Kürze eines Editorials werde ich mich auf die Behandlung von Patienten mit schweren psychischen Erkrankungen konzentrieren, d.h. der Kerngruppe von psychiatrischen Patienten mit oft psychotischen und andauernden Störungen, für die einst die Anstalten erbaut und später die Psychiatriereformen initiiert wurden.

In der Zeit zwischen 1952 und 1982 wurden Neuroleptika (einschließlich Clozapin), Antidepressiva und Lithium in die Psychiatrie eingeführt. Wesentliche psychotherapeutische Schulen wurden gegründet, wie die klientenzentrierte Gesprächstherapie, die kognitive Therapie, die lösungsorientierte Therapie und die Familientherapie. Die therapeutische Gemeinschaft als soziotherapeutischer Ansatz wurde entwickelt (hier dehne ich die Generation um wenige Jahre), und Gruppentherapien mit und ohne kreative Aktivitäten wurden eingeführt. Tageskliniken fanden ihren Weg nach Westeuropa, und multidisziplinäre Teams, die mit aufsuchendem Ansatz psychisch Kranke in der Gemeinde betreuen, wurden in ersten kontrollierten Studien positiv evaluiert. Für die Evaluation der Behandlungen fanden psychometrische Methoden Eingang in die Psychiatrie. Es wurden Skalen wie die „Brief Psychiatric Rating Scale“ oder „Hamilton Rating Scale for Depression“ entwickelt und randomisierte kontrollierte Studien wurden zum Goldstandard des Effektivitätsnachweises auch in der Psychiatrie. Und schließlich erlebte die Generation auch die Entschlüsse zu großen Psychiatriereformen, wie der Psychiatrieenquete in Deutschland und dem Gesetz 180 in Italien. Nicht schlecht für eine Generation, und entsprechend war die Begeisterung für und in der Psychiatrie groß.

Wie schneidet dagegen die letzte Generation ab eine Generation, in der weltweit Milliarden in die psychiatrische Forschung gesteckt wurden, und die sogar die offizielle „decade of the brain“ (die 1990er) beinhaltete? Es gibt kein einziges neues Medikament, das deutlich wirksamer wäre als die früher zur Verfügung stehenden (eventuelle Vorteile in Nebenwirkungsprofilen sind sicher wichtig, erhöhen aber nicht die Wirksamkeit). Die bedeutsamen psychotherapeutischen Therapieschulen und selbst die Evaluationsskalen sind im Wesentlichen immer noch die gleichen, und die Weiterentwicklung von soziotherapeutischen Methoden ist nicht sehr vorangekommen.

Mag die Bilanz der letzten 30 Jahre insgesamt schon nicht überragend ausfallen, haben die letzten 5-10 Jahre noch einmal spezifische Gründe geliefert, eher enttäuscht zu sein: Neuere Studien und Analysen haben gezeigt, dass alle Hoffnungen auf eine größere Wirksamkeit atypischer Neuroleptika (eventuell abgesehen vom Clozapin) unerfüllt blieben, dass Antidepressiva wahrscheinlich nur bei schweren Depressionen überhaupt einen Effekt haben, und dass mögliche positive Effekte von Frühbehandlungen psychotischer Störungen nicht von langer Dauer sind [1]. Zudem werden die Effekte von kognitiver Verhaltenstherapie in der Schizophreniebehandlung zunehmend skeptisch beurteilt [2]. Natürlich mag man all dem entgegenhalten, dass es ja große Fortentwicklungen in grundlagenorientierter Forschung, in genetischen Analysen und Bildgebung gegeben habe oder dass solche Fortschritte zumindest bald zu erwarten seien, und dass es 
nur eine Frage der Zeit sei, bis sich dies auch in neuen und effektiveren Therapiemethoden niederschlage. Hoffentlich ist dem so, aber zum Zeitpunkt, an dem dieses Editorial verfasst wird, ist ein solcher Durchbruch eben noch nicht gelungen, und die Therapiemöglichkeiten sind zur Zeit nicht viel anders als vor 30 Jahren. Also in einer ganzen Generation keinerlei Fortschritt? Doch, die Versorgung der meisten Patienten mit schweren psychischen Störungen hat sich zumindest in Westeuropa ganz entscheidend verbessert. 1982 waren die Psychiatriereformen zwar bereits initiiert, aber noch nicht umgesetzt. Seither haben sich Versorgungsstrukturen und -ansätze zum Teil grundlegend verändert. Vor allem haben massive Investitionen in bessere Einrichtungen und zusätzliches Personal dazu geführt, dass mehr Menschen mit psychischen Erkrankungen von Versorgungsdiensten erreicht werden und bessere Behandlungen erhalten. Die letzte Generation hat die sog. Enthospitalisierung von vielen vormals langzeithospitalisierten Patienten erlebt. Dies hat die Lebensqualität der meisten Patienten verbessert, und - anders als etwa in Teilen der USA sind nicht zahlreiche psychisch kranke Patienten obdachlos geworden oder im Gefängnis gelandet (obwohl immer noch zu viele). Psychiatrische Einrichtungen haben nicht nur mehr Personal per Patient, sondern das Personal (z. B. klinische Psychologen) ist in der Regel auch deutlich besser ausgebildet als früher.

Die Verbesserung der Versorgungsqualität wird vielen Zeitzeugen als offensichtlich erscheinen, aber gibt es auch wissenschaftliche Belege dafür, dass Behandlungen über die Zeit wirklich effektiver geworden sind? Kontrollierte Versuche, in denen ähnliche Patienten randomisierten Behandlungen in unterschiedlichen Jahrzehnten zugeführt werden, um die Effekte zu vergleichen, sind naturgemäß nicht möglich. Dennoch gibt es vielleicht einige indirekte Hinweise dafür, dass Behandlungsergebnisse allgemein jetzt besser sein könnten als vor ca. 30 Jahren. In randomisierten kontrollierten Studien wird in der Regel eine definierte spezifische Therapie mit einer Kontrollgruppe verglichen, die lediglich eine Standardbehandlung (,treatment as usual“) und bei Medikamentenversuchen zusätzlich auch noch ein Placebo erhält. Nun ist es über die Zeit immer schwerer geworden, in solchen Untersuchungen einen Effekt einer spezifischen Behandlung zu finden, d.h. einen Effekt, der über die Wirkung der Standard- oder Normalbehandlung hinausgeht. Dies wurde für die Neuroleptikabehandlung mit dem Hinweis diskutiert, dass halt der „Placeboeffekt“ so zugenommen habe, dass der zusätzliche Effekt eines Medikaments kaum noch nachzuweisen sei [3]. Auch bei jüngeren Studien, die den Ansatz des sog. „Assertive Outreach“ (intensiv aufsuchende Teams in der Gemeinde) oder der „Early Intervention“ (Frühinterventionen bei Patienten mit psychotischen Störungen) untersuchten, zeigte sich nur ein geringer oder noch häufiger gar kein Vorteil dieser neuen und intensiveren Behandlungen gegenüber den in England zur Normalversorgung gehörenden gemeindepsychiatrischen Teams (z.B. [4]). In der Literatur entspannte sich daraufhin eine Diskussion über die Gründe. Vergleiche zwischen den jüngeren und älteren Studien (die häufig aus den USA stammten und in denen die Versorgung in der Kontrollgruppe keinen modernen Ansprüchen genügte) zeigten, dass die Veränderungen in den Experimentalgruppen ähnlich waren. Patienten in der Standardbehandlung hatten aber so viel positivere Ergebnisse als in früheren Studien, dass eben kaum noch ein Unterschied wenn überhaupt - mit der neuen Intervention auszumachen war [5]. Worauf sind diese besseren Ergebnisse in den Kontrollgruppen zurückzuführen? Obwohl die Veränderungen in den Kontrollgruppen oft als Placeboeffekt bezeichnet werden, ist ein tatsächlicher Effekt der Einnahme eines Placebos unwahrscheinlich.
Es gibt kaum einen Nachweis für einen wirklichen Placeboeffekt über längere Zeiträume in psychiatrischen Behandlungen [6]. Es bleiben somit zwei Erklärungsmöglichkeiten: Entweder die Spontanverläufe psychischer Erkrankungen sind positiver als früher möglich, aber wenig einleuchtend -, oder aber Standardbehandlungen sind effektiver geworden. Die zweite Möglichkeit würde bedeuten, dass die verschiedenen zur Routine gehörenden Behandlungsfaktoren besser genutzt werden als zu früheren Zeiten, und ein wesentlicher Teil dieser Faktoren ist wahrscheinlich nicht spezifisch im Sinne der existierenden Theorien für die Wirkung medikamentöser, psychologischer oder sozialer Interventionen, sondern eher unspezifisch, also bisher ohne „spezifische“ Theorie zu ihrer Wirksamkeit. Dazu gehören die Effekte einer guten therapeutischen Beziehung, einer allgemeinen sozialen Unterstützung, unterschiedlicher psychologischer Hilfen außerhalb formaler Psychotherapie und eines hilfreichen therapeutischen Milieus.

Dieser enorme praktische Fortschritt ist weniger von einer wissenschaftlichen Sozialpsychiatrie als von einer sozialen Psychiatrie als gesundheitspolitischer Bewegung vorangetrieben worden. Er ist mit gesellschaftlichen Wertvorstellungen über die Integration von psychisch kranken und behinderten Menschen verbunden und einem politischen Willen, Geld für die Versorgung psychisch Kranker auszugeben, auch wenn sich diese Investition oft nicht in höherer Produktivität niederschlägt.

Wenn der Fortschritt in der Psychiatrie weniger auf gesicherten wissenschaftlichen Erkenntnissen als auf politischem Gestaltungswillen beruht, dann kann er in einem veränderten politischen Kontext auch leicht wieder verloren gehen. Es bleibt somit eine Aufgabe der Psychiatrie, sich dafür einzusetzen, dass die Werte der Psychiatriereformen und die erreichten Fortschritte nicht abhanden kommen - ein schwieriger Auftrag, wenn die politisch-gesellschaftlichen Rahmenbedingungen in Richtung von Kommerzialisierung und zunehmender sozialer Ungleichheit weisen. Außerdem mag diese Aufgabe für junge Psychiater auch nur begrenzt attraktiv sein. Es ist sicher leichter, sich für zukünftige Neuerungen und Reformen zu engagieren, als sich für die Verteidigung von Strukturen zu begeistern, die von einer früheren Generation errungen worden sind.

Für die wissenschaftliche Forschung ergibt sich die Forderung nach mehr Innovation, mehr Kreativität und gegebenenfalls auch sog. High-risk-Studien, die neue Ideen verfolgen und neue Methoden erproben. Die gezielte Untersuchung von bisher als unspezifisch betrachteten Behandlungsfaktoren könnte dabei ein lohnendes Feld sein.

\section{Literatur}

1 Bertelsen M, Jeppesen P, Petersen L et al. Five-year follow-up of a randomized multicenter trial of intensive early intervention vs standard treatment for patients with a first episode of psychotic illness: the OPUS trial. Arch Gen Psychiatry 2008; 65: 762 - 771

2 Jones C, Hacker D, Meaden A et al. Cognitive behaviour therapy versus other psychosocial treatments for schizophrenia. Cochrane Database Syst Rev 2011; 4: CD00524

$3 \mathrm{Kemp} A \mathrm{~S}$, Schooler NR, Kalili AH et al. What is causing the reduced drugplacebo difference in recent schizophrenia clinical trials and what can be done about it? Schizophr Bull 2010; 36: 504-509

4 Killaspy $H$, Bebbington P, Blizard $P$ et al. The REACT study: randomised evaluation of assertive community treatment in north London. BMJ 2006; 332: 815-820

5 Burns T, Catty J, Watt $H$ et al. International differences in home treatment for mental health problems: Results of a systematic review. $\mathrm{Br} J$ Psychiatry 2002; 181: 375-382

6 Hróbjartsson A, Gøtzsche PC. Is the placebo powerless? An analysis of clinical trials comparing placebo with no treatment N Engl J Med 2001; 344: $1594-1602$ 